\title{
The Effects of Habitual Aquatic Walking on Arterial Stiffness and Body Composition in Postmenopausal Women: A Cross-Sectional Study
}

\author{
Won-Mok Son PhD, Jung-Jun Park PhD \\ Division of Sports Science, Pusan National University, Busan, Korea
}

PURPOSE: Menopause is associated with increased carotid-femoral pulse wave velocity (cfPWV), an indicator of arterial stiffness, which is an independent marker of cardiovascular risk. This study aimed to investigate the cfPWV, blood pressure, and body composition with habitual exercise for at least 1 year in postmenopausal women performing habitual aquatic walking versus age-matched sedentary postmenopausal and premenopausal women.

METHODS: A total of 115 postmenopausal and premenopausal women were divided into the following groups: (1) postmenopausal women performing habitual aquatic walking (HAW; $n=57)$, (2) sedentary postmenopausal women (SPO; $n=41)$, and (3) sedentary premenopausal women (SPR, $n=17$ ). The HAW group participated 3 days per week, 60 minutes per day, for at least 1 year, whereas the SPO and SPR groups performed no regular exercise, for less than 2 days per week, 30 minutes per day, for at least 1 year. The cfPWV, BP, and body composition were assessed in all groups.

RESULTS: The cfPWV $(p<.05)$, augmentation pressure $(p<.05)$, and DBP were significantly lower in the SPR group than in the SPO group.AIx@75, BMI, \%BF, and SBP were significantly lower in the SPR group than in the SPO and HAW groups. Lean body mass was higher in the SPR group than in the SPO and HAW groups $(p<.05)$. The cfPWV, AP, AIx@75, SBP, and \%BF were lower in the HAW group than in the SPO group $(p<.05)$. Furthermore, the cfPWV was significantly correlated with aging and SBP.

CONCLUSIONS: This cross-sectional study indicates that sedentary postmenopausal women may be at a higher risk of cardiovascular disease than postmenopausal women who participate in habitual aquatic walking.

Key words: Arterial stiffness, Aquatic walking, Blood pressure, Cardiovascular disease, Menopause

\section{INTRODUCTION}

Aging is a major risk factor for cardiovascular disease (CVD), and a sedentary lifestyle in older adulthood increases the incidence of CVD in this population [1,2]. Menopause is a natural aging process and is associated with an increased risk of vascular dysfunction and developing CVD. Furthermore, it increases arterial stiffness due to reduced estrogen and elastin levels [3]. CVD is the second leading cause of death in Korean women aged 65 years or older [4]. CVD is caused by a variety of factors, but one of the key contributors is increased arterial stiffness [5]. The main methods to measure arterial stiffness are pulse wave velocity
(PWV) and the augmentation index (AI) [6]. Zaydun et al. [7] reported that the incidence of arterial stiffness is higher in postmenopausal women. Increased arterial stiffness reduces baroreflex sensitivity, which in turn has an adverse impact on blood pressure (BP) regulation [8]. The consequent increase in systolic BP leads to increased pulse pressure and left ventricular loading, which elevates the risk of CVD [9].

Regular exercise has been reported to improve microcirculatory vascular function that is responsible for reduced cardiovascular risk in postmenopausal women [10]. Aquatic exercise is a whole-body exercise that utilizes the principle of buoyancy, and it is an ideal exercise for older adults with weakened joints [11]. We have previously shown that aquatic

Corresponding author: Jung-Jun Park Tel +82-51-510-2713 Fax+82-51-510-3747 E-mail jjparkpnudpusan.ac.kr

Keywords Arterial stiffness, Aquatic walking, Blood pressure, Cardiovascular disease, Menopause

Received 2 Apr 2021 Revised 28 May 2021 Accepted 31 May 2021

@)This is an Open Access article distributed under the terms of the Creative Commons Attribution Non-Commercial License (https://creativecommons.org/licenses/by-nc/4.0/) which permits unrestricted non-commercial use, distribution, and reproduction in any medium, provided the original work is properly cited. 
walking exercise for four 60 minutes sessions per week for 12 weeks is effective in improving ambulation in patients with peripheral artery disease [12]. This improvement was determined by important metrics, including leg PWV and BP. In addition, a previous study reported that after a single session aquatic exercise, older women with hypertension had reduced systolic BP (SBP) and diastolic BP (DBP) [13].

While the positive outcomes of aquatic and other exercise regimens have been well documented, no cross-sectional study has investigated the effects of regular exercise and sedentary lifestyle on arterial stiffness, BP, and body composition of postmenopausal women. The aim of this study was to examine these physiological parameters in postmenopausal women who performed habitual aquatic walking exercises for at least 1 year in comparison to a sedentary lifestyle of age-matched participants. We hypothesized that the habitual aquatic walking participants would exhibit decreased arterial stiffness and BP as well as a healthier body composition, compared to the sedentary lifestyle participants.

\section{METHODS}

\section{Participants}

Participants were recruited using flyers from churches and multiple sports centers in South Korea. In this cross-sectional study, a total of 115 women comprised the following groups: 1) habitual aquatic walking postmenopausal women group (HAW; $\mathrm{n}=57$ ), 2) sedentary postmenopausal women group (SPO; $\mathrm{n}=41$ ), and 3) sedentary premenopausal women group (SPR, $\mathrm{n}=17$ ) (Table 1). The HAW group were recruited from multiple sports centers and exercised 3 days per week, 60 minutes per day for at least 1 year. The SPO and SPR groups were recruited from churches and did not exercise regularly (i.e., exercised less than 2 days per week, 30 minutes per day in the previous year). All participants provided written informed consent before their inclusion in the study.

\section{Body composition}

Height (to the nearest $0.1 \mathrm{~cm}$ ) was measured using a stadiometer (DS102, Jenix, Korea). Body composition was measured using bioelectrical impedance analysis (BIA) (InBody 720, Biospace, Korea), and comprised assessment of body mass (to the nearest $0.1 \mathrm{~kg}$ ), percentage body fat (\%BF; to the nearest $0.1 \%$ ), and lean body mass (to the nearest $0.1 \mathrm{~kg}$ ). Body mass index (BMI) was calculated as follows: BMI = body mass ( $\mathrm{kg}) /$ height $\left(\mathrm{m}^{2}\right)$.

\section{Blood pressure}

All measurements were performed in a noise-free laboratory $\left(25 \pm 1^{\circ} \mathrm{C}\right)$ in the morning (9:00-11:00 AM). Resting SBP and DBP were measured using an automated sphygmomanometer (BP-203RVIII; Matsunaga Sokki Co., Ltd., Japan) in the seated position after a 5-minute rest period. The average of two BP measurements was recorded as the resting BP Resting BP measurements were obtained in accordance with JNC-7 guidelines [14]. The sphygmomanometer cuff was fastened on the right arm, with the lower cuff margin approximately $2 \mathrm{~cm}$ above the elbow crease and the arrow on the cuff aligned with the brachial artery.

\section{Carotid-femoral pulse wave velocity (cfPWV)}

cfPWV was measured using a thigh cuff, carotid tonometry, and a commercially available applanation tonometer (SphygmoCor, AtCor Medical Ltd., Australia) pre-installed with analysis software (version 8.0,

Table 1. Participant characteristics

\begin{tabular}{|c|c|c|c|c|c|c|}
\hline & $\operatorname{SPR}(A)(n=17)$ & $\mathrm{SPO}(B)(n=41)$ & $\mathrm{HAW}(\mathrm{C})(\mathrm{n}=57)$ & $F$-value & Post-hoc & $t$-value \\
\hline Age (yr) & $37.1 \pm 2.1$ & $71.1 \pm 5.1$ & $69.7 \pm 6.0$ & & & \\
\hline Height (cm) & $164.7 \pm 5.1$ & $153.4 \pm 5.6$ & $154.0 \pm 5.2$ & & & \\
\hline Mass (kg) & $60.3 \pm 10.0$ & $60.9 \pm 6.4$ & $58.5 \pm 6.5$ & & & \\
\hline $\mathrm{BMI}\left(\mathrm{kg} / \mathrm{m}^{2}\right)$ & $22.2 \pm 3.3$ & $25.8 \pm 2.4$ & $24.7 \pm 2.7$ & $10.917^{* * * *}$ & $A<B, C$ & -1.681 \\
\hline Body fat (\%) & $26.6 \pm 5.9$ & $37.1 \pm 4.2$ & $32.8 \pm 5.3$ & $27.144^{* * *}$ & $A<B, C$ & $-3.992^{++\dagger}$ \\
\hline lean body mass $(\mathrm{kg})$ & $24.5 \pm 4.4$ & $20.4 \pm 2.1$ & $21.1 \pm 1.8$ & $17.303^{* * * *}$ & $A<B, C$ & 1.847 \\
\hline Systolic BP (mmHg) & $123.5 \pm 12.8$ & $140.1 \pm 11.3$ & $133.5 \pm 10.1$ & $14.194^{* * *}$ & $A<B, C$ & $-3.201^{\dagger+}$ \\
\hline Diastolic BP (mmHg) & $76.2 \pm 13.9$ & $83.2 \pm 7.4$ & $80.4 \pm 8.1$ & $3.741^{*}$ & $A<B$ & -1.5 \\
\hline $\operatorname{cfPWV}(\mathrm{m} / \mathrm{s})$ & $7.2 \pm 1.0$ & $8.7 \pm 1.2$ & $7.8 \pm 1.2$ & $11.038^{* * *}$ & $A<B$ & $-3.307^{\dagger+}$ \\
\hline $\mathrm{AP}(\mathrm{mmHg})$ & $9.8 \pm 3.6$ & $17.8 \pm 6.8$ & $12.8 \pm 6.9$ & $11.356^{* * * *}$ & $A<B$ & $-3.162^{t+}$ \\
\hline Alx@75 (\%) & $17.4 \pm 5.9$ & $30.0 \pm 7.9$ & $24.6 \pm 9.4$ & $13.910^{* * * *}$ & $A<B, C$ & $-2.833^{\dagger \dagger}$ \\
\hline
\end{tabular}

Data are mean $\pm S D$.

SPE, sedentary premenopausal women; SPO, sedentary postmenopausal women; HAW, habitual aqua walking postmenopausal women. ${ }^{*} p<.05,{ }^{* * *} p<.001$ different than SPO and HAW. ${ }^{+\dagger} p<.01,{ }^{+t+} p<.001$ different than SPO. 
SphygmoCor Cardiovascular Management Suite). cfPWV was calculated as the distance between the manubrium sternum to the femoral artery minus the distance between the carotid artery and the manubrium sternum divided by the CF time interval. PWV was calculated using the following formula: $\mathrm{PWV}=\mathrm{L}$ (distance) $/ \Delta \mathrm{t}$ (time) $(\mathrm{m} / \mathrm{s})[15]$.

\section{Augmentation pressure (AP), and augmentation index at heart rate (HR) 75 (Alx@75)}

Augmentation pressure (AP) was defined as the difference between the second and first systolic peaks. AIx was measured as the augmentation pressure expressed as a percentage of aortic pulse pressure (calculated directly using Pulse Wave Analysis software). AIx was calculated using the following formula: $\mathrm{AIx}=100 \times((\mathrm{P} 2-\mathrm{P} 1) /($ Pulse Pressure $))$. AIx was normalized to a heart rate of 75 beats/minutes (AIx@75) because it is influenced by heart rate. Two measurements, acquired at 10-second intervals, were averaged [16].

\section{Statistical analysis}

All data are presented as mean \pm standard deviation. Statistical analyses were performed with one-way ANOVA with least significant differences post-hoc analyses used to determine differences between specific groups by SPSS 25.0 version software (SPSS Science, Chicago, IL, USA) analysis. Pearson correlation was used to correlate aging and arterial stiffness, and SBP and arterial stiffness. Data (mean \pm SD) was considered statistically significant at a value of $p<.05$.

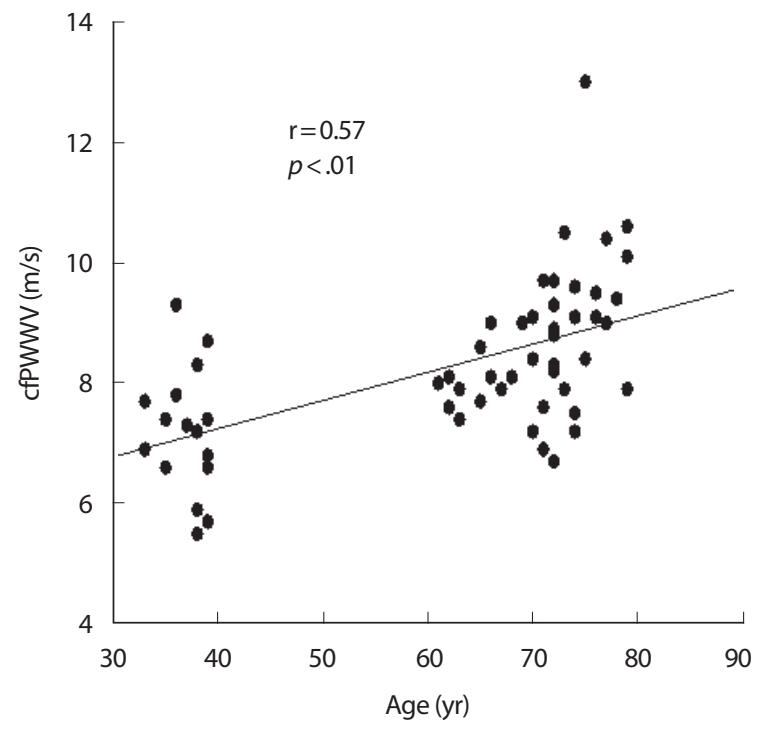

Fig. 1. Correlation of cfPWV and age.

\section{RESULTS}

cfPWV was lower in the SPR group than in the SPO group $(p<.001)$, while cfPWV in the HAW group was lower than that in the SPO group $(p<.01)$

AP was lower in the SPR group than in the SPO group $(p<.001)$, while AP in the HAW group was lower than that in the SPO group $(p<.01)$. AIx@75 was lower in the SPR group than in the SPO and HAW groups $(p<.001)$, while AIx@75 in the HAW group was lower than that in the SPO group $(p<.01)$.

SBP was lower in the SPR group than in the SPO and HAW groups $(p<.001)$, while SBP in the HAW group was lower than that in the SPO group $(p<.01)$. DBP was lower in the SPR group than in the SPO group $(p<.05)$.

BMI was lower in the SPR group than in the SPO and HAW groups $(p<.001) . \%$ BF was lower in the SPR group than in the SPO and HAW groups $(p<.001)$, while \%BF in the HAW group was lower than that in the SPO group $(p<.001)$. LBM was higher in the SPR group than in the SPO and HAW groups $(p<.001)$.

We observed that cfPWV correlated with age $(\mathrm{r}=0.57, p<.01)$ and SBP $(\mathrm{r}=0.52, p<.01)$ (Figs. 1, 2).

\section{DISCUSSION}

\section{Arterial stiffness}

Menopause with aging is a major cause of increased arterial stiffness

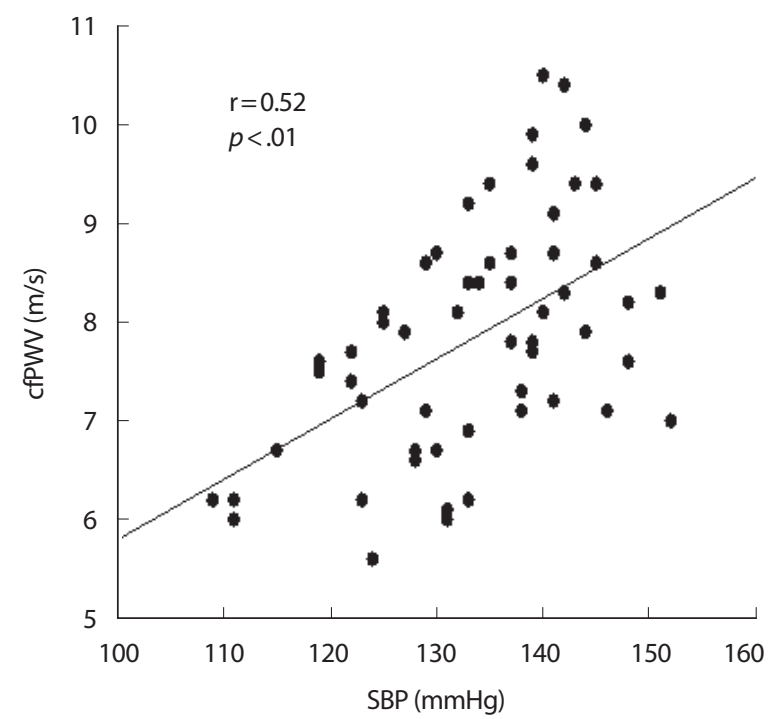

Fig. 2. Correlation of cfPWV and SBP. 
[7]. Aging causes structural changes in the arterial wall that reduces the vasodilation capacity and increases blood flow velocity [17]. In particular, menopause is known to reduce elastin and estrogen concentrations, thereby accelerating arterial stiffness [18]. Previous studies have demonstrated an increase in arterial stiffness and AIx in postmenopausal women [19].

However, exercise is known to improve the risk factors for cardiovascular disease, and previous studies have suggested that cardiovascular functions can be improved in older adults by promoting cardiovascular adaptation through regular aerobic exercise [20]. Matsubara et al. [21] showed that an aerobic exercise program decreased arterial stiffness in menopausal women. One potential mechanism underlying exercise-induced reduction in arterial stiffness is known to involve increased endovascular shear stress experienced by blood vessel walls during exercise [22]. Shear stress leads to increased secretion and bioavailability of nitric oxide (NO), a vasodilator, which subsequently improves vascular endothelial cell function and reduces vascular resistance and tone [23]. Additionally, a previous study suggested that a decreased BP is associated with improved arterial stiffness [24]. In the present study, the HAW group showed lower cfPWV, AP, and AIx@75 than the SPO group, and there was a correlation of cfPWV with age and BP. Our results support that notion that arterial stiffness increases due to menopause with aging, and that regular aquatic walking exercise for 1 year or longer may improve arterial stiffness in these women. However, we did not directly examine the vasodilation markers and endothelial function in response to an exercise program. Therefore, further studies evaluating the vasodilation markers and endothelial function are warranted.

\section{Blood pressure}

Menopause is a known high-risk factor for CVD and is associated with estrogen deficiency in postmenopausal women, causing vascular dysfunction, eventually resulting in increased BP [18]. A previous study reported that increased SBP contributes to CVD risk in older adults [25].

However, another study reported that reduced SBP decreased CVDassociated mortality in middle-aged and older adults [26]. Aerobic exercise is known to improve BP [27], and Forcier et al. [28], in their metaanalysis of 33 studies, demonstrated that individuals who perform high aerobic fitness had significantly reduced SBP and DBP. Furthermore, Arca et al. [29] documented that aquatic walking exercise decreased BP in menopausal women with hypertension.

Reduction in SBP and DBP by regular aerobic exercise, including re- duced vascular resistance and vascular tone in the peripheral arteries by improved endothelial function, may be a potential mechanism [30]. Another known mechanism is decreased sympathetic drive due to the reduction of $\alpha$ - and $\beta$-adrenergic receptors of sympathetic neurotransmitters [31]. This cross-sectional study showed that the HAW group had lower BP than the SPO group. Our findings indicate that habitual aquatic walking exercise can play a significant role in reducing BP in postmenopausal women. However, the mechanism underlying this reduction in BP was not examined in this study. Therefore, future studies should explore the mechanism involved.

\section{Body composition}

Menopause is associated with a decrease in physiological and metabolic functions, weakening of skeletal muscles, and increase in fat mass [32]. In particular, a reduction of estrogen in postmenopausal women is associated with an increase in their subcutaneous fat mass and abdominal obesity [33]. Notably, abdominal obesity elevates the risk of insulin resistance and arteriosclerosis, and it directly impacts the prevalence of CVD $[34,35]$. Increased abdominal obesity in menopause is more evident among women than similar-aged men [36]. Therefore, increased abdominal obesity is a prominent concern for the health of older women.

However, regular physical activity is considered as the most effective treatment for obesity in older adults [37], and has been shown to mitigate obesity-related metabolic disorders by decreasing body weight [38] and body fat, thereby facilitating metabolic functions by increasing lean mass [39]. Lambert et al. [40] previously demonstrated that aquatic treadmill exercise reduced body weight and \%BF in adult men and women, and Bergamin et al. [41] substantiated that aquatic walking exercise improved body composition in older men and women. In the present study, the HAW group showed a lower \%BF than the SPO group. We confirmed that regular participation in aquatic walking exercises for 1 year or longer decreased \%BF. Therefore, regular exercise may contribute to the effective treatment of obesity in older adults.

\section{CONCLUSION}

The results of this cross-sectional study showed that menopause with aging is associated with increased cfPWV, AIx@75, and BP and that regular aquatic walking exercises are linked to improvements in arterial stiffness and BP in postmenopausal women. However, we did not directly examine the vasodilation markers and the mechanism underlying 
$\mathrm{BP}$ reduction due to aquatic exercise programs. Additionally, we did not examine other potential factors that affected arterial stiffness and BP (e.g., daily physical activity level, nutritional intake, etc.). Therefore, future studies should investigate the potential mechanisms underlying exercise-induced vasodilation and BP reduction, as well as ascertain the influence of other potential factors.

\section{CONFLICT OF INTEREST}

No potential conflict of interest relevant to this article.

\section{AUTHOR CONTRIBUTIONS}

Conceptualization: WM Son; Data curation: WM Son ; Formal analysis: WM Son, JJ Park; Funding acquisition: WM Son, JJ Park; Methodology: WM Son, JJ Park; Project administration: WM Son, JJ Park; Visualization: WM Son, JJ Park; Writing-original draft: WM Son, JJ Park; Writing review \& editing: WM Son, JJ Park.

\section{ORCID}

$\begin{array}{ll}\text { Won-Mok Son } & \text { https://orcid.org/0000-0001-8942-6515 } \\ \text { Jung Jun Park } & \text { https://orcid.org/0000-0002-2518-7225 }\end{array}$

\section{REFERENCES}

1. Lakatta EG, Levy D. Arterial and cardiac aging: major shareholders in cardiovascular disease enterprises: part I: aging arteries: a "set up" for vascular disease. Circulation. 2003;107(1):139-46.

2. Ferrari AU, Radaelli A, Centola M. Invited review: aging and the cardiovascular system. J Appl Physiol (1985). 2003;95(6):2591-7.

3. Pearson TA, Blair SN, Daniels SR, Eckel RH, Fair JM, et al. AHA guidelines for primary prevention of cardiovascular disease and stroke: 2002 update: consensus panel guide to comprehensive risk reduction for adult patients without coronary or other atherosclerotic vascular diseases. American Heart Association Science Advisory and Coordinating Committee. Circulation. 2002;106(3):388-91.

4. Shin H-Y, Kim J, Lee S, Park MS, Park S, et al. Cause-of-death statistics in 2018 in the republic of korea. Journal of the Korean Medical Association/Taehan Uisa Hyophoe Chi. 2020;63(5).

5. Chambers CV, Markson L, Diamond JJ, Lasch L, Berger M. Health beliefs and compliance with inhaled corticosteroids by asthmatic patients in primary care practices. Respir Med. 1999;93(2):88-94.

6. Tomiyama H, Yamashina A. Non-invasive vascular function tests: their pathophysiological background and clinical application. Circ J. 2010;74(1):24-33.

7. Zaydun G, Tomiyama H, Hashimoto H, Arai T, Koji Y, et al. Menopause is an independent factor augmenting the age-related increase in arterial stiffness in the early postmenopausal phase. Atherosclerosis. 2006;184(1):137-42.

8. Mattace-Raso FU, van den Meiracker AH, Bos WJ, van der Cammen TJ, Westerhof BE, et al. Arterial stiffness, cardiovagal baroreflex sensitivity and postural blood pressure changes in older adults: the rotterdam study. J Hypertens. 2007;25(7):1421-6.

9. Laurent S, Cockcroft J, Van Bortel L, Boutouyrie P, Giannattasio C, et al. Expert consensus document on arterial stiffness: methodological issues and clinical applications. Eur Heart J. 2006;27(21):2588-605.

10. Alkhatib A, Klonizakis M. Effects of exercise training and Mediterranean diet on vascular risk reduction in post-menopausal women. Clin Hemorheol Microcirc. 2014;57(1):33-47.

11. Sherlock L, Fournier S, DeVallance E, Lee K, Carte S, et al. Effects of shallow water aerobic exercise training on arterial stiffness and pulse wave analysis in older individuals. International Journal of Aquatic Research and Education. 2014;8(4):3.

12. Park SY, Wong A, Son WM, Pekas EJ. Effects of heated water-based versus land-based exercise training on vascular function in individuals with peripheral artery disease. J Appl Physiol (1985). 2020;128(3):56575.

13. Cunha RM, Macedo CB, Araujo SF, Santos JC, Borges VS, et al. Subacute blood pressure response in elderly hypertensive women after a water exercise session: a controlled clinical trial. High Blood Press Cardiovasc Prev. 2012;19(4):223-7.

14. Chobanian AV, Bakris GL, Black HR, Cushman WC, Green LA, et al The seventh report of the joint national committee on prevention, detection, evaluation, and treatment of high blood pressure: the JNC 7 report. JAMA. 2003;289(19):2560-72.

15. Dagdeviren C, Su Y, Joe P, Yona R, Liu Y, et al. Conformable amplified lead zirconate titanate sensors with enhanced piezoelectric response for cutaneous pressure monitoring. Nat Commun. 2014;5:4496.

16. Wilkinson IB, MacCallum H, Flint L, Cockcroft JR, Newby DE, et al. The influence of heart rate on augmentation index and central arterial pressure in humans. J Physiol. 2000;525 Pt 1:263-70. 
17. Giallauria F, Ling SM, Schreiber C, Maggio M, Shetty V, et al. Arterial stiffness and bone demineralization: the baltimore longitudinal study of aging. Am J Hypertens. 2011;24(9):970-5.

18. Suzuki H, Kondo K. Pulse wave velocity in postmenopausal women. Pulse. (Basel) 2013;1(1):4-13.

19. London GM, Guerin AP, Pannier B, Marchais SJ, Stimpel M. Influence of sex on arterial hemodynamics and blood pressure. Role of body height. Hypertension. 1995;26(3):514-9.

20. Mora S, Cook N, Buring JE, Ridker PM, Lee IM. Physical activity and reduced risk of cardiovascular events: potential mediating mechanisms. Circulation. 2007;116(19):2110-8.

21. Matsubara T, Miyaki A, Akazawa N, Choi Y, Ra SG, et al. Aerobic exercise training increases plasma klotho levels and reduces arterial stiffness in postmenopausal women. Am J Physiol Heart Circ Physiol. 2014;306(3):H348-55.

22. Hellstrom G, Fischer-Colbrie W, Wahlgren NG, Jogestrand T. Carotid artery blood flow and middle cerebral artery blood flow velocity during physical exercise. J Appl Physiol (1985). 1996;81(1):413-8.

23. Walther C, Gielen S, Hambrecht R. The effect of exercise training on endothelial function in cardiovascular disease in humans. Exerc Sport Sci Rev. 2004;32(4):129-34.

24. Seals DR, Tanaka H, Clevenger CM, Monahan KD, Reiling MJ, et al. Blood pressure reductions with exercise and sodium restriction in postmenopausal women with elevated systolic pressure: role of arterial stiffness. J Am Coll Cardiol. 2001;38(2):506-13.

25. Psaty BM, Furberg CD, Kuller LH, Bild DE, Rautaharju PM, et al. Traditional risk factors and subclinical disease measures as predictors of first myocardial infarction in older adults: the cardiovascular health study. Arch Intern Med. 1999;159(12):1339347.

26. Hall WD. Risk reduction associated with lowering systolic blood pressure: review of clinical trial data. Am Heart J. 1999;138(3 Pt 2):225-30.

27. Seals DR, Silverman HG, Reiling MJ, Davy KP. Effect of regular aerobic exercise on elevated blood pressure in postmenopausal women. Am J Cardiol. 1997;80(1):49-55.

28. Forcier K, Stroud LR, Papandonatos GD, Hitsman B, Reiches M, et al. Links between physical fitness and cardiovascular reactivity and recovery to psychological stressors: a meta-analysis. Health Psychol. 2006;25
(6):723-39

29. Arca EA, Martinelli B, Martin LC, Waisberg CB, Franco RJ. Aquatic exercise is as effective as dry land training to blood pressure reduction in postmenopausal hypertensive women. Physiother Res Int. 2014;19 (2):93-8.

30. Park SY, Ives SJ, Gifford JR, Andtbacka RH, Hyngstrom JR, et al. Impact of age on the vasodilatory function of human skeletal muscle feed arteries. Am J Physiol Heart Circ Physiol. 2016;310(2):H217-25.

31. Brownley KA, Hinderliter AL, West SG, Girdler SS, Sherwood A, et al Sympathoadrenergic mechanisms in reduced hemodynamic stress responses after exercise. Med Sci Sports Exerc. 2003;35(6):978-86.

32. Carr MC. The emergence of the metabolic syndrome with menopause. J Clin Endocrinol Metab. 2003;88(6):2404-11.

33. Lizcano F, Guzman G. Estrogen deficiency and the origin of obesity during menopause. Biomed Res Int. 2014;2014:757461.

34. Albu JB, Kovera AJ, Johnson JA. Fat distribution and health in obesity. Ann N Y Acad Sci. 2000;904:491-501.

35. Despres JP, Lemieux I, Bergeron J, Pibarot P, Mathieu P, et al. Abdominal obesity and the metabolic syndrome: contribution to global cardiometabolic risk. Arterioscler Thromb Vasc Biol. 2008;28(6):1039-49.

36. Toth MJ, Matthews DE, Tracy RP, Previs MJ. Age-related differences in skeletal muscle protein synthesis: relation to markers of immune activation. Am J Physiol Endocrinol Metab. 2005;288(5):E883-91.

37. Bouchonville MF, Villareal DT. Sarcopenic obesity: how do we treat it? Curr Opin Endocrinol Diabetes Obes. 2013;20(5):412-9.

38. Mougios V, Kazaki M, Christoulas K, Ziogas G, Petridou A. Does the intensity of an exercise programme modulate body composition changes? Int J Sports Med. 2006;27(3):178-81.

39. Venables MC, Jeukendrup AE. Physical inactivity and obesity: links with insulin resistance and type 2 diabetes mellitus. Diabetes Metab Res Rev. 2009;25 Suppl 1:S18-23.

40. Lambert BS, Greene NP, Carradine AT, Joubert DP, Fluckey JD, et al. Aquatic treadmill training reduces blood pressure reactivity to physical stress. Med Sci Sports Exerc. 2014;46(4):809-16.

41. Bergamin M, Ermolao A, Tolomio S, Berton L, Sergi G, et al. Waterversus land-based exercise in elderly subjects: effects on physical performance and body composition. Clin Interv Aging. 2013;8:1109-17. 